\title{
THE ABELL 35-TYPE PLANETARY NUCLEI
}

\author{
HOWARD E. BOND \\ Space Telescope Science Institute \\ Baltimore, Maryland USA \\ ROBIN CIARDULLO \\ Pennsylvania State University \\ University Park, Pennsylvania USA \\ and \\ MICHAEL G. MEAKES \\ Space Telescope Science Institute \\ Baltimore, Maryland USA
}

The nuclei of the low-surface-brightness PNe A 35, LoTr 1, and LoTr 5 are binaries containing rapidly rotating late-type subgiants or giants and extremely hot $\left(T_{\text {eff }} \gtrsim 100,000 \mathrm{~K}\right)$ companions detected by the $I U E$ satellite. All three objects show low-amplitude, periodic photometric variations in the optical band (with periods of 0.76 or $3.3,6.6$, and 5.9 days, respectively).

Since the photometric amplitudes change on timescales of weeks to months, we attribute the photometric variations to starspots on the cool components. The periodicities thus correspond to the rotational periods of the cool stars, and the true orbital periods remain unknown. However, for the very similar object HD 128220 , which does not lie within a planetary nebula, the orbital period is known to be 872 days, suggesting that the orbital periods could be quite long.

The evolutionary origin of these systems remains puzzling, but we speculate that they have recently emerged from a common-envelope interaction in which the cool main-sequence component was spun up to rapid rotation, and still remains out of thermal equilibrium. 